$\cdot$ Review

\title{
Role of autophagy in the pathogenesis of multiple sclerosis
}

\author{
Peizhou Liang ${ }^{1,2}$, Weidong Le ${ }^{2}$ \\ ${ }^{1}$ Institute of Neuroscience, Shanghai Institutes for Biological Sciences, Chinese Academy of Sciences, Shanghai 200031, \\ China \\ ${ }^{2}$ Center for Translational Research on Neurological Diseases, the First Affiliated Hospital, Dalian Medical University, Dalian \\ 116011, China
}

Corresponding author: Weidong Le. E-mail: wdle@sibs.ac.cn

(C) Shanghai Institutes for Biological Sciences, CAS and Springer-Verlag Berlin Heidelberg 2015

Autophagy plays an important role in maintaining the cellular homeostasis. One of its functions is to degrade unnecessary organelles and proteins for energy recycling or amino-acids for cell survival. Ablation of autophagy leads to neurodegeneration. Multiple sclerosis (MS), a permanent neurological impairment typical of chronic inflammatory demyelinating disorder, is an auto-immune disease of the central nervous system (CNS). Autophagy is tightly linked to the innate and adaptive immune systems during the autoimmune process, and several studies have shown that autophagy directly participates in the progress of MS or experimental autoimmune encephalomyelitis (EAE, a mouse model of MS). Dysfunction of mitochondria that intensively influences the autophagy pathway is one of the important factors in the pathogenesis of MS. Autophagy-related gene (ATG) 5 and immune-related GTPase M (IRGM) 1 are increased, while ATG16L2 is decreased, in T-cells in EAE and active relapsing-remitting MS brains. Administration of rapamycin, an inhibitor of mammalian target of rapamycin (mTOR), ameliorates relapsing-remitting EAE. Inflammation and oxidative stress are increased in MS lesions and EAE, but Lamp2 and the LC3-II/LC3-I ratio are decreased. Furthermore, autophagy in various glial cells plays important roles in regulating neuro-inflammation in the CNS, implying potential roles in MS. In this review, we discuss the role of autophagy in the peripheral immune system and the CNS in neuroinflammation associated with the pathogenesis of MS.

Keywords: autophagy; multiple sclerosis; neuro-inflammation

\section{Introduction}

Autophagy, a lysosome-dependent degradation pathway, contributes to maintaining cellular homeostasis. Clearance of long-lived proteins, unnecessary organelles, and aggregate-prone proteins is mainly executed by autophagy for recycling cellular materials ${ }^{[1]}$. There are three subtypes of autophagy, macroautophagy, chaperone-mediated autophagy (CMA), and mitophagy. Macroautophagy is the major type for selective degradation of protein aggregates or misfolded proteins. The ubiquitin-labeled proteins are recognized by autophagy receptors, such as $\mathrm{p} 62$, neighbor of BRCA1 gene 1 (NBR1), and NIP3-like protein X (NIX), to form autophagosomes that then fuse with lysosomes for degradation. Many autophagy-related genes function during this process, such as Unc51-like kinase (ULK1) and autophagy-related gene (ATG) 5. Mammalian target of rapamycin (mTOR) represses autophagy by regulating ULK1 phosphorylation, while AMPK activates this process. CMA mediates the degradation of large molecular-weight proteins containing the KFERQ motif recognized by heat shock cognate protein of $70 \mathrm{kDa}$ (HSC70). The substrate is then escorted to lysosome-associated membrane protein 2 (LAMP2A) for lysosomal degradation. Mitophagy is responsible for the degradation of damaged mitochondria. Parkin and PINK1 play critical roles in this process ${ }^{[2]}$. More 
attention has been paid to autophagy in the pathogenesis of neurodegenerative diseases, such as Alzheimer's disease $(A D)^{[3]}$, Parkinson's disease $(P D)^{[4]}$ and amyotrophic lateral sclerosis (ALS) ${ }^{[5]}$.

Multiple sclerosis (MS) is a non-inherited neurodegenerative disease characterized by the involvement of innate and adaptive immunity. MS occurs mainly in young adults and its incidence in females is two to three times higher than that in males. About $85 \%$ of MS patients show relapse-remission before irreversible progression. It is widely accepted that MS is an auto-immune disease caused by autoreactive T-cells that target the myelin sheath in the central nervous system $(\mathrm{CNS})^{[6,7]}$. These myelin sheath-targeting T-cells infiltrate the CNS via breakdown of the blood brain barrier, attack the myelin sheath, and initiate chronic inflammatory responses, leading to the loss of axons and neurons. The fact that adoptive transfer of activated myelin-specific $\mathrm{CD} 4^{+} \mathrm{T}$-cells can induce experimental autoimmune encephalomyelitis (EAE) demonstrates that auto-immunity is indispensable in the pathogenesis of $\mathrm{MS}^{[8]}$. Genome-wide association studies have shown that immunologically-relevant genes, especially T-helper-cell differentiation genes, are significantly overrepresented in the pathogenesis of $\mathrm{MS}^{[9]}$. However, it is still unclear what exactly causes the disease. One of the initiators of MS is antigen from virus or bacteria that is similar to the myelin basic protein peptide ${ }^{[10,11]}$. The autoimmunity hypothesis is now being challenged since the finding that, like many other neurodegenerative disorders, gray matter lesions and brain atrophy are detectable before MS onset ${ }^{[12,13]}$. So the initiators of MS still need further investigation. An important factor in MS development is neuro-inflammation, which is also one of the main characteristics of MS (chronic inflammation, infiltration of peripheral immune cells, demyelination, transected axons, and gliosis). The frail neurons die when they are exposed to the inflammatory environment. Pro-inflammatory factors such as IL17 impair the differentiation of oligodendrocyte progenitor cells (OPCs) in $\mathrm{MS}^{[14]}$.

Autophagy is tightly linked with auto-immune diseases, including $\mathrm{MS}^{[15-17]}$. The extracellular matrix molecule fibronectin aggregates in chronically demyelinating MS and the relapsig phase of EAE; it may be resistant to degradation and affect the remyelination process ${ }^{[18]}$. Soluble oligomers have been detected in the brain and cerebral spinal fluid of MS patients ${ }^{[19]}$. Meanwhile, oxidized protein aggregates increase in EAE, probably due to the reduced autophagy level ${ }^{[20]}$. Moreover, autophagy intensively regulates inflammation in various diseases. To unravel the potential role of autophagy in the pathogenesis of MS, we highlight its possible importance in both the generation of auto-reactive lymphocytes and the regulation of neuro-inflammation. Differential cell-type specificity of autophagy in the peripheral immune system and the CNS might modulate the onset and progression of MS (Fig. 1).

\section{Autophagy and Mitochondria in MS}

Mitochondria play important roles in general and selective autophagic flux, including mitophagy. Dysfunction of mitochondria during stress is one of the important factors involved in the pathogenesis of MS and EAE. Autophagy maintains the architecture of mitochondria, restores their function, and prevents their dysfunction, which are critical for cell survival and the pathogenesis of $\mathrm{MS}^{[21-23]}$. In MS, the decreased expression of cytochrome $c$ oxidase subunit $5 b$ may impair the function of mitochondria ${ }^{[24]}$. Dysfunction of mitochondria produces reactive oxygen species (ROS), which contribute to demyelination and axonal loss ${ }^{[25]}$. Autophagy clears depolarized mitochondria to reduce the excessive production of ROS by increasing BECN1 or regulating ATG4 activity ${ }^{[26,27]}$, which is protective in MS.

\section{Autophagy and MS in the Peripheral Immune System Mutual Regulation of Autophagy and Inflammation}

The connections between autophagy and inflammation are complex. Each regulates the other by different mechanisms. Pattern-recognition receptors for pathogen recognition, such as toll-like receptors (TLRs) and NOD-like receptors (NLRs) can elicit autophagy for pathogen clearance ${ }^{[28]}$. However, autophagy negatively regulates inflammation to prevent the harmful amplification of inflammatory factors. For instance, ATG9 suppresses the stimulator of IFN genes protein (STING) in type-I IFN signaling to reduce the inflammatory response ${ }^{[29]}$. Blockade of autophagy leads to the activation of inflammasomes which control the proteolytic processing and secretion of IL-1 $1 \beta$ and IL-18 under inflammatory stress ${ }^{[30,31]}$. Also, ablation of autophagyrelated 16-like 1 (ATG16L1) increases the production of 


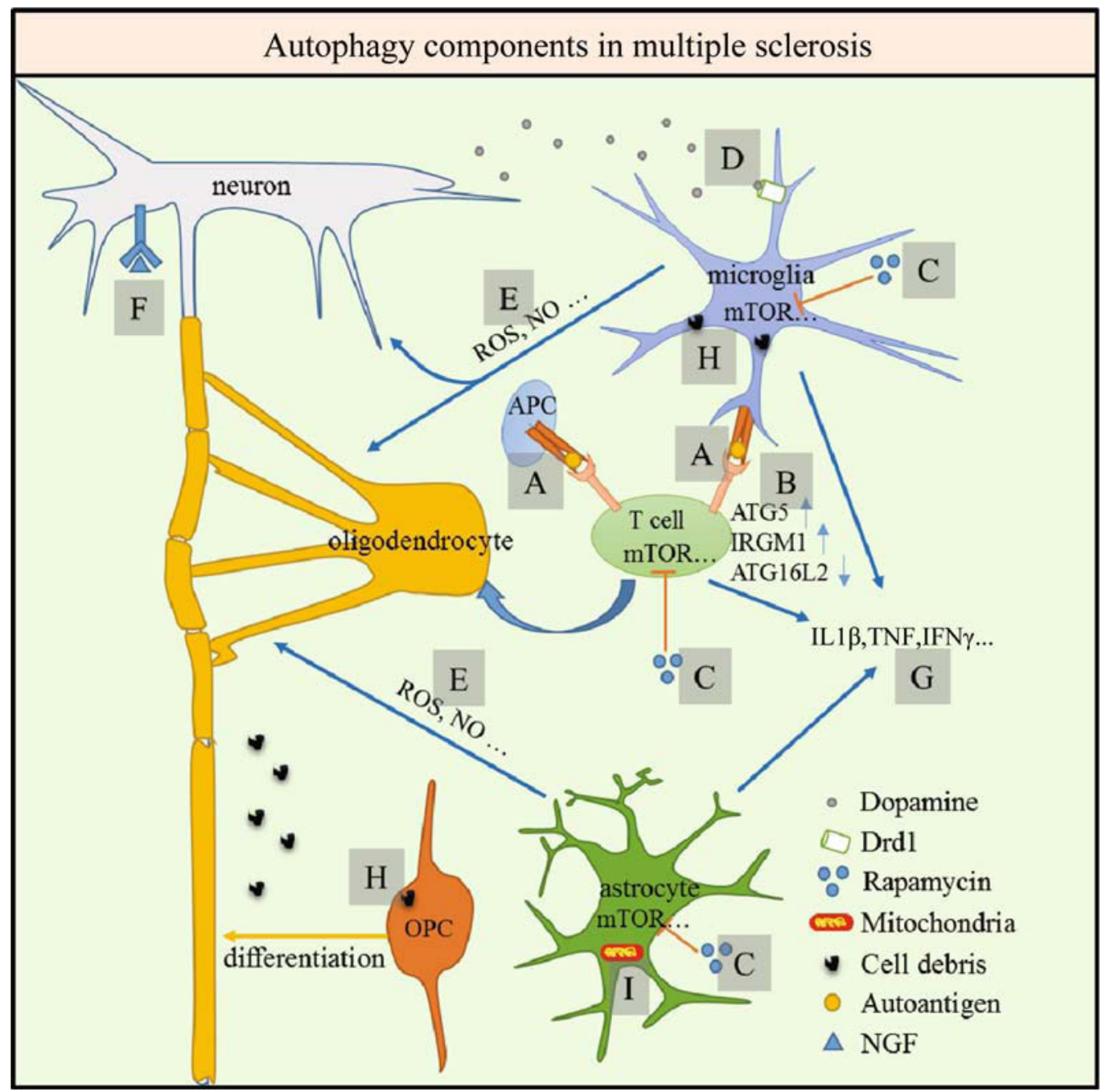

Fig. 1. Autophagy in the pathogenesis of multiple sclerosis. A: Autophagy is indispensable for antigen presentation in antigen presenting cells (APC, e.g. thymic epithelial cells, microglia). B: In MS patients and EAE mice, ATG5 and IRGM1 increase in T-cells to enhance their survival. Downregulation of ATG16L2 may perturb the homeostasis of T-cells. C: Rapamycin, the inhibitor of mTOR that represses autophagy, regulates inflammation and benefits EAE. D: Drd1 in microglia or macrophages activated by dopamine triggers autophagy to inhibit inflammasome activation during neuro-inflammation. E: ROS and NO produced by microglia and astrocytes in neuro-inflammation regulate autophagy in various cell types, including astrocytes. F: In MS patients, NGF increases in cerebrospinal fluid and represses autophagy in neurons to prevent cell death. G: Pro-inflammatory factors, such as IL-1 $1 \beta$, TNF, and IFNy, can induce autophagy in macrophages or microglia. H: Cell debris or protein aggresomes in MS or EAE are removed by microglia or macrophages through phagocytosis or autophagy. OPCs may also clear cellular debris and protein aggresomes by endocytosis and autophagy. I: Autophagy is necessary to maintain the architecture and restore the function of mitochondria in astrocytes during neuro-inflammation. Depolarized mitochondria are also removed by autophagy.

IL-1 $\beta$ and IL-18 in the mouse model of Crohn's disease ${ }^{[32]}$. Moreover, infection of ATG5-deficient myeloid cells with $M$. tuberculosis increases the expression of IL-17, a potent mediator in autoimmunity ${ }^{[33]}$. In primary effective T-cells, p62 serves as a receptor for autophagic degradation of ubiquitinated BCL10 during TCR activation to protect cells from excessively activated NF-KB pathway ${ }^{[34]}$.

Inflammatory factors in turn regulate the progress of autophagy. The T-helper 1 (Th1) cell cytokine IFNy induces autophagy in macrophages to suppress the survival of mycobacteria ${ }^{[35]}$. Another pro-inflammatory factor, Tnf, also induces autophagy in monocytes/macrophages ${ }^{[36]}$. 
However, the Th2 cell cytokines IL-4 and IL-13 ablate autophagy in macrophages ${ }^{[36]}$.

\section{Autoreactive T-Cell Regulation by Autophagy}

Besides innate immunity, autophagy also participates intensively in shaping the adaptive immune system. Th1 cells are crucial at the early stage of MS pathogenesis, and Th17 cells are indispensable in the later stage. Autophagy is necessary for the generation of autoreactive T-cells. It participates in multiple functions of the adaptive immune system, such as antigen presentation and maintenance of the homeostasis of T-cells.

Autophagy mediates antigen-presentation during the generation of autoreactive T-cells. Autophagy enhances antigen-presentation to $\mathrm{CD} 4^{+} \mathrm{T}$-cells via major histocompatibility complex class II molecules ${ }^{[37,38]}$. In thymic epithelial cells, autophagy contributes to the positive and negative control of the T-cell repertoire, playing a critical role in the elimination of self-reactive T-cells and exotic pathogens. Mice thymi with ATG5 ablation show infiltration of autoreactive $\mathrm{CD}^{+} \mathrm{T}$-cells into multiple organs and induce autoimmune colitis similar to Crohn's diseaserelated phenotypes ${ }^{[38]}$. Atg7 ablation in dendritic cells (DCs), the most potent antigen-presenting cells, ameliorates EAE. Blockade of autophagy in DCs by chloroquine also delays the onset or reduces the severity of $E A E^{[39]}$. DCs from patients with Crohn's disease with ATG16L 1 and NOD2 variants are defective in antigen presentation ${ }^{[40]}$. The presentation of citrullinated peptides by DCs, macrophages, and thymic DCs is blocked by inhibition of autophagy with 3-methyladenine ${ }^{[41]}$.

Autophagy regulates the homeostasis, proliferation, differentiation, maturation, and apoptosis of T-cells. It also maintains the calcium flux and the pro-survival process and counteracts pro-apoptotic action to maintain T-cell homeostasis $^{[42-45]}$. Cyt1 induces autophagosome formation when it binds to CD46, which may function in maintaining the homeostasis of T-cells during adaptive immunity ${ }^{[46]}$. Moreover, the expression of ATG16L2 in T-cells is reduced, and this is likely to perturb the homeostasis of T-cells in $\mathrm{MS}^{[47]}$. ATG5 plays important roles in T-cell survival and controls the proliferation, differentiation and maturation of CD4/8 $8^{+}$T-cells and B-cells ${ }^{[48-50]}$. mTOR-deficient T-cells fail to differentiate into T-helper cells, Th1, Th2, and Th17 effector cells ${ }^{[51,52]}$. ATG5 is significantly elevated in T-cells in MS lesions and EAE mice compared to controls. The increase of autophagy in T-cells may promote their survival, contributing to the pathogenesis of $\mathrm{MS}^{[53]}$. Immune-related GTPase M1 is highly expressed in MS lesions in the CNS and EAE models, and contributes to the pathogenesis of MS by increasing the survival of autoreactive CD4 ${ }^{+}$ $\mathrm{T}_{\text {-cells }}{ }^{[17]}$. Rapamycin, an inhibitor of $\mathrm{mTOR}$, ameliorates the relapsing-remitting EAE (RR-EAE) in SJL/j mice by increasing the Treg cell population ${ }^{[54]}$. However, the contributions of autophagy in different T-cell populations to cell death depend on the context. Upon activation of autophagy, Th2 cells undergo growth factor-withdrawalmediated cell death ${ }^{[55]}$. The induction of autophagy through binding of HIV-1 envelope glycoproteins to CXC chemokine receptor 4 of $\mathrm{CD}^{+}$T-cells is necessary for apoptosis ${ }^{[56]}$. FADD or casp8 in T-cells limits autophagy and protects against cell necrosis and inflammation ${ }^{[57,58]}$. Alteration of the proportions of different $\mathrm{T}$-cell groups affects the immune response ${ }^{[59]}$.

\section{Pathological Role of Autophagy in MS}

\section{Autophagy and Neuro-inflammation}

CNS autophagy is linked with serious neurodegenerative diseases. Deficiency of basal autophagy in neurons leads to protein aggregation and finally neurodegenerative diseases ${ }^{[60,61]}$. Neuro-inflammation is one of the important factors that cause neurodegenerative diseases, especially MS. Autophagy and neuro-inflammation regulate each other to affect the progression of various diseases in the CNS.

Neuro-inflammation is negatively regulated by autophagy to avoid its harm to the CNS. Microglia, resident immune cells in the CNS, play important roles in the initiation and sustenance of neuro-inflammation to clear pathogens. They produce pro-inflammatory factors and neurotoxic factors including ROS and nitric oxide (NO). Subsequently-activated astrocytes further amplify the inflammatory response to produce more proinflammatory factors, chemokines, and NO, which have toxic effects on primary neurons ${ }^{[7,62]}$. However, autophagy plays an essential role in maintaining neuro-inflammation at a harmless level. Activation of Drd1 by dopamine inhibits NOD-LRR-containing pyrin domain 3 (NLRP3) inflammasome activation through autophagic degradation 
of NLRP3 via the E3 ubiquitin ligase $\mathrm{MARCH} 7^{[63]}$. The dopamine system also regulates lymphocyte activity in lupus and MS, suggesting an important role of dopamine in the regulation of peripheral or CNS infiltrated lymphocytes during the pathogenesis of $\mathrm{MS}^{[64,65]}$. In addition, inhibition of neuro-inflammation by autophagy has great benefits on ischemia ${ }^{[66]}$. Autophagy may be a stress response to the negative feedback regulation of neuro-inflammation. In the chronic LPS model, pro-inflammatory factor IL-1 $\beta$ in cortex is positively correlated with the autophagy markers Beclin-1 and LC3-II and inversely correlated with p62 ${ }^{[67]}$.

Autophagy can be deleterious depending on the disease. Activation of the autophagic pathway in ischemia leads to the death of astrocytes and neurons, which can be rescued by autophagy inhibitors ${ }^{[68,69]}$. Meanwhile, blockade of microglial autophagy in permanent middle cerebral artery occlusion by autophagy inhibitors reduces the severity of the disease ${ }^{[70]}$. Thus, autophagy in different diseases and cell types in the CNS seems to function differently. Autophagic cell death is still under debate and needs further investigation ${ }^{[7]}$.

\section{Autophagy in Glial Cells}

Until recently, most studies on autophagy in the CNS focused on neurons, with less on glial cells. The activation of glial cells plays important roles in neuro-inflammation, and these cells are indispensable for the development of $\mathrm{MS}^{[7]}$. MS progression occurs between glial activation and gliosis. Autophagy in various glial cells may play a role in responding to stress in MS. Therefore, it is urgent to study the effect of glial autophagy on neurological diseases, including MS.

Microglia are the first line of defense in the CNS, monitoring exotic and intrinsic pathogens. During demyelination, cell debris is mainly phagocytized by microglia or microphages, a process that requires autophagy-related genes ${ }^{[72]}$. Impairment of autophagy in microglia may hinder debris clearance, leading to damaged remyelination and augmentation of persistent neuroinflammation. The rapid activation of microglia produces pro-inflammatory cytokines and free radicals, and acts as the major antigen-presenting cell ${ }^{[73,74]}$. Pro-inflammatory factors such as IL-1 $\beta$ can trigger microglial autophagy, suggesting links between autophagy and inflammation activation ${ }^{[75]}$. mTOR plays a pivotal role in autophagy suppression and promotes inflammation in microglia, and rapamycin may have a beneficial effect in the treatment of $\mathrm{MS}^{[76]}$. mTOR inhibitors reduce neuro-inflammation by inhibiting microglial activation or viability, decreasing proinflammatory cytokines ${ }^{[77]}$. Simian immunodeficiency virusinfected microglia attenuate the survival of neurons by inhibiting neuronal autophagy ${ }^{[78]}$, which implicates a cellcell type regulation of autophagy in the CNS. However, microglial autophagy in middle cerebral artery occlusion makes the disease worse ${ }^{[70]}$. It is possible that the role of microglial autophagy depends on the disease.

Astrocytes are the primary supportive elements for neuronal structure and neurotrophy, comprising $\sim 50 \%$ of cells in the adult mammalian brain. Autophagy is necessary for the differentiation and maturation of astrocytes ${ }^{[79]}$, and can be initiated in inflammatory stress and may be important in the development of MS. During pro-inflammatory stimuli, autophagy is induced to maintain mitochondrial networks ${ }^{[22]}$. Impairment of the autophagy pathway by ablation of Atg7 in astrocytes in the inflammatory environment amplifies the response to produce more neurotoxic factors such as ROS, which induces astrocytic cell death ${ }^{[22,80]}$. mTOR in the astrocyte may play a protective role in ischemia via its downstream kinase $\mathrm{S} 6 \mathrm{~K} 1^{[81]}$. mTOR can also regulate the stability of iNOS mRNA in astrocytes, reducing the neurotoxic effect of NO which impairs autophagy by disrupting the BECN1 complex and activates mTORC $1^{[82-84]}$.

OPCs are indispensable during oligodendrocyte differentiation and myelin remyelination; they are activated for remyelination in MS lesions ${ }^{[85,86]}$. They also play an important role in MS pathogenesis by modulating immune activity in the CNS. Act1 deletion in OPCs blocks the IL-17 pathway, attenuating neuro-inflammation and increasing OPC maturation ${ }^{[14]}$. OPCs can also clear $\beta$-amyloid peptides by triggering endocytosis and autophagy ${ }^{[87]}$, implying an autophagic role of OPCs in MS. However, the exact functions of OPCs in MS need further investigation, including those of autophagy and its differentiation, nutritional support, debris clearance, and inflammation regulation.

Roles of Autophagy in MS-Associated Demyelination and Remyelination

Autophagy is closely linked to demyelination and remyelination. It plays potential roles in improving 
Schwann cell remyelination in demyelinating peripheral neuropathies ${ }^{[88]}$. Rapamycin, a potent autophagy inducer, improves myelination, leading to enhanced neuronal survival in tuberous sclerosis ${ }^{[89]}$. Meanwhile, in Long-Evans shaker rats, an increased autophagy level increases the number of myelinated axons and myelin sheath thickness during dysmyelination and demyelination, which implies that the autophagy pathway is a direct target for therapy for demyelination ${ }^{[90]}$. Furthermore, high mobility group box chromosomal protein 1 (HMGB1), which promotes autophagy, is elevated in $M S$ and $E A E^{[91]}$. However, the progression of MS and EAE involves many cell types. Different cell types in the lesion site might show different patterns of autophagy. In MS, especially in the acute phase, nerve growth factor (NGF) is dramatically increased in the cerebrospinal fluid ${ }^{[92]}$. NGF inhibits autophagy and cell death of neurons through the p75 neurotrophin receptor, suggesting a protective role in $\mathrm{MS}^{[93]}$. In $\mathrm{MS}$ lesions, Lamp2 expression is reduced ${ }^{[94]}$, implying an impairment of autophagy. The mTOR signaling pathway that inhibits autophagy restores the regrowth of axons in the CNS, which is important for remyelination in $\mathrm{MS}^{[95,96]}$. Moreover, in acute and chronic EAE, protein aggresomes in the spinal cord and a reduced LC3-II/LC3-I ratio suggest that the protein turnover mechanism through autophagy is impaired ${ }^{[20]}$. In fact, the mechanism of autophagy in the pathogenesis of MS in the CNS is still superficially understood. Autophagy in different stages of the disease and different cell types needs further investigation to determine the causal relationship between autophagy and MS.

\section{Conclusive Remarks}

So far, little is known about the exact mechanism of autophagy in MS. It is still controversial whether autophagy leads to cell death in MS or is a rescue mechanism activated as part of an endogenous neuroprotective response, since autophagy plays opposite functions in inflammation and cell survival, depending on the context. Drugs modifying the immune system and remyelination have been used to help with relapse management, reduce the degree of disability, and improve the quality of life ${ }^{[97,98]}$. The effects of rapamycin and chloroquine in the therapy of EAE shed light on the autophagy pathway as a potential target for drug development. The inhibition of autophagy in mesenchymal stem cells provides a novel strategy to improve therapeutic effects by enhancing the suppression of $\mathrm{CD}^{+}{ }^{+} \mathrm{T}_{\text {-cells }}{ }^{[99]}$. In order to develop effective and more specific therapeutic strategies, we need to fully understand the complex interplay between autophagy and MS in different cell types.

\section{ACKNOWLEDGEMENTS}

This review was supported by a grant from the National Natural Science Foundation of China (81430021 and 81370470), China Postdoctoral Science Foundation (2014M551465) and the Collaborative Innovation Center for Brain Science.

Received date: 2015-05-20; Accepted date: 2015-07-05

\section{REFERENCES}

[1] Rubinsztein DC. The roles of intracellular protein-degradation pathways in neurodegeneration. Nature 2006, 443: 780-786.

[2] Nixon RA. The role of autophagy in neurodegenerative disease. Nat Med 2013, 19: 983-997.

[3] Nixon RA, Wegiel J, Kumar A, Yu WH, Peterhoff C, Cataldo $A$, et al. Extensive involvement of autophagy in Alzheimer disease: an immuno-electron microscopy study. J Neuropathol Exp Neurol 2005, 64: 113-122.

[4] Lynch-Day MA, Mao K, Wang K, Zhao M, Klionsky DJ. The role of autophagy in Parkinson's disease. Cold Spring Harb Perspect Med 2012, 2: a009357.

[5] Song CY, Guo JF, Liu Y, Tang BS. Autophagy and its comprehensive impact on ALS. Int J Neurosci 2012, 122: 695-703.

[6] Steinman L. Multiple sclerosis: a two-stage disease. Nat Immunol 2001, 2: 762-764.

[7] Glass CK, Saijo K, Winner B, Marchetto MC, Gage FH. Mechanisms underlying inflammation in neurodegeneration. Cell 2010, 140: 918-934.

[8] Ben-Nun A, Wekerle H, Cohen IR. The rapid isolation of clonable antigen-specific $\mathrm{T}$ lymphocyte lines capable of mediating autoimmune encephalomyelitis. Eur J Immunol 1981, 11: 195-199.

[9] International Multiple Sclerosis Genetics C, Wellcome Trust Case Control C, Sawcer S, Hellenthal G, Pirinen M, Spencer $\mathrm{CC}$, et al. Genetic risk and a primary role for cell-mediated immune mechanisms in multiple sclerosis. Nature 2011, 476: 214-219.

[10] Sospedra M, Martin R. Immunology of multiple sclerosis. Annu Rev Immunol 2005, 23: 683-747.

[11] Fujinami RS, Oldstone MB. Amino acid homology between 
the encephalitogenic site of myelin basic protein and virus: mechanism for autoimmunity. Science 1985, 230: 10431045.

[12] Stys PK, Zamponi GW, van Minnen J, Geurts JJ. Will the real multiple sclerosis please stand up? Nat Rev Neurosci 2012, 13: $507-514$.

[13] Ellwardt E, Zipp F. Molecular mechanisms linking neuroinflammation and neurodegeneration in MS. Exp Neurol 2014, 262 Pt A: 8-17.

[14] Kang Z, Wang C, Zepp J, Wu L, Sun K, Zhao J, et al. Act1 mediates IL-17-induced EAE pathogenesis selectively in NG2(+) glial cells. Nat Neurosci 2013, 16: 1401-1408.

[15] Wellcome Trust Case Control C. Genome-wide association study of 14,000 cases of seven common diseases and 3,000 shared controls. Nature 2007, 447: 661-678.

[16] Zhou XJ, Lu XL, Lv JC, Yang HZ, Qin LX, Zhao MH, et al. Genetic association of PRDM1-ATG5 intergenic region and autophagy with systemic lupus erythematosus in a Chinese population. Ann Rheum Dis 2011, 70: 1330-1337.

[17] Xu H, Wu ZY, Fang F, Guo L, Chen D, Chen JX, et al. Genetic deficiency of Irgm1 (LRG-47) suppresses induction of experimental autoimmune encephalomyelitis by promoting apoptosis of activated CD4+ T cells. FASEB J 2010, 24: 1583-1592.

[18] Stoffels JM, de Jonge JC, Stancic M, Nomden A, van Strien $\mathrm{ME}, \mathrm{Ma} \mathrm{D}$, et al. Fibronectin aggregation in multiple sclerosis lesions impairs remyelination. Brain 2013, 136: 116-131.

[19] David MA, Tayebi M. Detection of protein aggregates in brain and cerebrospinal fluid derived from multiple sclerosis patients. Front Neurol 2014, 5: 251.

[20] Dasgupta A, Zheng J, Perrone-Bizzozero NI, Bizzozero OA. Increased carbonylation, protein aggregation and apoptosis in the spinal cord of mice with experimental autoimmune encephalomyelitis. ASN Neuro 2013, 5: e00111.

[21] Kalman B, Laitinen K, Komoly S. The involvement of mitochondria in the pathogenesis of multiple sclerosis. $J$ Neuroimmunol 2007, 188: 1-12.

[22] Motori E, Puyal J, Toni N, Ghanem A, Angeloni C, Malaguti $\mathrm{M}$, et al. Inflammation-induced alteration of astrocyte mitochondrial dynamics requires autophagy for mitochondrial network maintenance. Cell Metab 2013, 18: 844-859.

[23] Lu Q, Zhang J, Allison R, Gay H, Yang WX, Bhowmick NA, et al. Identification of extracellular delta-catenin accumulation for prostate cancer detection. Prostate 2009, 69: 411-418.

[24] Broadwater L, Pandit A, Clements R, Azzam S, Vadnal J, Sulak $M$, et al. Analysis of the mitochondrial proteome in multiple sclerosis cortex. Biochim Biophys Acta 2011, 1812: 630-641.

[25] van Horssen J, Witte ME, Schreibelt G, de Vries HE. Radical changes in multiple sclerosis pathogenesis. Biochim Biophys
Acta 2011, 1812: 141-150.

[26] Chen Y, Gibson SB. Is mitochondrial generation of reactive oxygen species a trigger for autophagy? Autophagy 2008, 4: 246-248.

[27] Chen Y, McMillan-Ward E, Kong J, Israels SJ, Gibson SB. Oxidative stress induces autophagic cell death independent of apoptosis in transformed and cancer cells. Cell Death Differ 2008, 15: 171-182.

[28] Saitoh T, Akira S. Regulation of innate immune responses by autophagy-related proteins. J Cell Biol 2010, 189: 925-935.

[29] Saitoh T, Fujita N, Hayashi T, Takahara K, Satoh T, Lee H, et al. Atg9a controls dsDNA-driven dynamic translocation of STING and the innate immune response. Proc Natl Acad Sci U S A 2009, 106: 20842-20846.

[30] Deretic V, Saitoh T, Akira S. Autophagy in infection, inflammation and immunity. Nat Rev Immunol 2013, 13: 722-737.

[31] Shi CS, Shenderov K, Huang NN, Kabat J, Abu-Asab M, Fitzgerald KA, et al. Activation of autophagy by inflammatory signals limits IL-1beta production by targeting ubiquitinated inflammasomes for destruction. Nat Immunol 2012, 13: 255263.

[32] Saitoh T, Fujita N, Jang MH, Uematsu S, Yang BG, Satoh $\mathrm{T}$, et al. Loss of the autophagy protein Atg16L1 enhances endotoxin-induced IL-1 beta production. Nature 2008, 456: 264-268.

[33] Castillo EF, Dekonenko A, Arko-Mensah J, Mandell MA, Dupont $\mathrm{N}$, Jiang $\mathrm{S}$, et al. Autophagy protects against active tuberculosis by suppressing bacterial burden and inflammation. Proc Natl Acad Sci U S A 2012, 109: E31683176.

[34] Paul S, Kashyap AK, Jia W, He YW, Schaefer BC. Selective autophagy of the adaptor protein Bcl10 modulates $\mathrm{T}$ cell receptor activation of NF-kappaB. Immunity 2012, 36: 947958.

[35] Gutierrez MG, Master SS, Singh SB, Taylor GA, Colombo $M I$, Deretic V. Autophagy is a defense mechanism inhibiting BCG and Mycobacterium tuberculosis survival in infected macrophages. Cell 2004, 119: 753-766.

[36] Harris J, Keane J. How tumour necrosis factor blockers interfere with tuberculosis immunity. Clin Exp Immunol 2010, 161: 1-9.

[37] Paludan C, Schmid D, Landthaler M, Vockerodt M, Kube D, Tuschl T, et al. Endogenous MHC class II processing of a viral nuclear antigen after autophagy. Science 2005, 307: 593-596.

[38] Nedjic J, Aichinger M, Emmerich J, Mizushima N, Klein L. Autophagy in thymic epithelium shapes the T-cell repertoire and is essential for tolerance. Nature 2008, 455: 396-400.

[39] Bhattacharya A, Parillon X, Zeng S, Han S, Eissa NT. 
Deficiency of autophagy in dendritic cells protects against experimental autoimmune encephalomyelitis. J Biol Chem 2014, 289: 26525-26532.

[40] Cooney R, Baker J, Brain O, Danis B, Pichulik T, Allan P, et al. NOD2 stimulation induces autophagy in dendritic cells influencing bacterial handling and antigen presentation. Nat Med 2010, 16: 90-97.

[41] Ireland JM, Unanue ER. Autophagy in antigen-presenting cells results in presentation of citrullinated peptides to CD4 T cells. J Exp Med 2011, 208: 2625-2632.

[42] Jia W, Pua HH, Li QJ, He YW. Autophagy regulates endoplasmic reticulum homeostasis and calcium mobilization in T Iymphocytes. J Immunol 2011, 186: 1564-1574.

[43] Lee JS, Li Q, Lee JY, Lee SH, Jeong JH, Lee HR, et al. FLIPmediated autophagy regulation in cell death control. Nat Cell Biol 2009, 11: 1355-1362.

[44] $\mathrm{He} M X, \mathrm{He} Y W$. A role for $\mathrm{C}-\mathrm{FLIP}(L)$ in the regulation of apoptosis, autophagy, and necroptosis in T lymphocytes. Cell Death Differ 2013, 20: 188-197.

[45] Hubbard VM, Valdor R, Patel B, Singh R, Cuervo AM, Macian F. Macroautophagy regulates energy metabolism during effector T cell activation. J Immunol 2010, 185: 7349-7357.

[46] Ni Choileain S, Astier AL. CD46 plasticity and its inflammatory bias in multiple sclerosis. Arch Immunol Ther Exp (Warsz) 2011, 59: 49-59.

[47] Yin L, Liu J, Dong H, Xu E, Qiao Y, Wang L, et al. Autophagyrelated gene16L2, a potential serum biomarker of multiple sclerosis evaluated by bead-based proteomic technology. Neurosci Lett 2014, 562: 34-38.

[48] Miller BC, Zhao Z, Stephenson LM, Cadwell K, Pua HH, Lee HK, et al. The autophagy gene ATG5 plays an essential role in B lymphocyte development. Autophagy 2008, 4: 309-314.

[49] Pua HH, He YW. Maintaining T lymphocyte homeostasis: another duty of autophagy. Autophagy 2007, 3: 266-267.

[50] Pua HH, Dzhagalov I, Chuck M, Mizushima N, He YW. A critical role for the autophagy gene Atg5 in T cell survival and proliferation. J Exp Med 2007, 204: 25-31.

[51] Delgoffe GM, Kole TP, Zheng Y, Zarek PE, Matthews KL, Xiao $B$, et al. The mTOR kinase differentially regulates effector and regulatory $\mathrm{T}$ cell lineage commitment. Immunity 2009, 30: 832-844.

[52] Chi $\mathrm{H}$. Regulation and function of mTOR signalling in $\mathrm{T}$ cell fate decisions. Nat Rev Immunol 2012, 12: 325-338.

[53] Alirezaei M, Fox HS, Flynn CT, Moore CS, Hebb AL, Frausto RF, et al. Elevated ATG5 expression in autoimmune demyelination and multiple sclerosis. Autophagy 2009, 5: 152-158.

[54] Esposito M, Ruffini F, Bellone M, Gagliani N, Battaglia M, Martino G, et al. Rapamycin inhibits relapsing experimental autoimmune encephalomyelitis by both effector and regulatory $T$ cells modulation. J Neuroimmunol 2010, 220: 52-63.

[55] Li C, Capan E, Zhao Y, Zhao J, Stolz D, Watkins SC, et al. Autophagy is induced in CD4+ T cells and important for the growth factor-withdrawal cell death. J Immunol 2006, 177: 5163-5168.

[56] Espert L, Denizot M, Grimaldi M, Robert-Hebmann V, Gay B, Varbanov $\mathrm{M}$, et al. Autophagy is involved in T cell death after binding of HIV-1 envelope proteins to CXCR4. J Clin Invest 2006, 116: 2161-2172.

[57] Bell BD, Leverrier S, Weist BM, Newton RH, Arechiga AF, Luhrs KA, et al. FADD and caspase- 8 control the outcome of autophagic signaling in proliferating T cells. Proc Natl Acad Sci U S A 2008, 105: 16677-16682.

[58] Yu L, Alva A, Su H, Dutt P, Freundt E, Welsh S, et al. Regulation of an ATG7-beclin 1 program of autophagic cell death by caspase-8. Science 2004, 304: 1500-1502.

[59] Oreja-Guevara C, Ramos-Cejudo J, Aroeira LS, Chamorro B, Diez-Tejedor E. TH1/TH2 Cytokine profile in relapsingremitting multiple sclerosis patients treated with Glatiramer acetate or Natalizumab. BMC Neurol 2012, 12: 95.

[60] Komatsu M, Waguri S, Chiba T, Murata S, Iwata J, Tanida $\mathrm{I}$, et al. Loss of autophagy in the central nervous system causes neurodegeneration in mice. Nature 2006, 441: 880884.

[61] Hara T, Nakamura K, Matsui M, Yamamoto A, Nakahara Y, Suzuki-Migishima R, et al. Suppression of basal autophagy in neural cells causes neurodegenerative disease in mice. Nature 2006, 441: 885-889.

[62] Saijo K, Winner B, Carson CT, Collier JG, Boyer L, Rosenfeld MG, et al. A Nurr1/CoREST pathway in microglia and astrocytes protects dopaminergic neurons from inflammationinduced death. Cell 2009, 137: 47-59.

[63] Yan Y, Jiang W, Liu L, Wang X, Ding C, Tian Z, et al. Dopamine controls systemic inflammation through inhibition of NLRP3 inflammasome. Cell 2015, 160: 62-73.

[64] Besser MJ, Ganor Y, Levite M. Dopamine by itself activates either D2, D3 or D1/D5 dopaminergic receptors in normal human T-cells and triggers the selective secretion of either IL10, TNFalpha or both. J Neuroimmunol 2005, 169: 161-171.

[65] Pacheco R, Contreras F, Zouali M. The dopaminergic system in autoimmune diseases. Front Immunol 2014, 5: 117.

[66] Zhou X, Zhou J, Li X, Guo C, Fang T, Chen Z. GSK-3beta inhibitors suppressed neuroinflammation in rat cortex by activating autophagy in ischemic brain injury. Biochem Biophys Res Commun 2011, 411: 271-275.

[67] Francois A, Terro F, Quellard N, Fernandez B, Chassaing D, Janet $\mathrm{T}$, et al. Impairment of autophagy in the central nervous system during lipopolysaccharide-induced inflammatory stress in mice. Mol Brain 2014, 7: 56. 
[68] Qin AP, Liu CF, Qin YY, Hong LZ, Xu M, Yang L, et al. Autophagy was activated in injured astrocytes and mildly decreased cell survival following glucose and oxygen deprivation and focal cerebral ischemia. Autophagy 2010, 6: 738-753.

[69] Wang JY, Xia Q, Chu KT, Pan J, Sun LN, Zeng B, et al. Severe global cerebral ischemia-induced programmed necrosis of hippocampal CA1 neurons in rat is prevented by 3-methyladenine: a widely used inhibitor of autophagy. $\mathrm{J}$ Neuropathol Exp Neurol 2011, 70: 314-322.

[70] Yang Z, Zhong L, Zhong S, Xian R, Yuan B. Hypoxia induces microglia autophagy and neural inflammation injury in focal cerebral ischemia model. Exp Mol Pathol 2015, 98: 219-224.

[71] Ghavami S, Shojaei S, Yeganeh B, Ande SR, Jangamreddy JR, Mehrpour M, et al. Autophagy and apoptosis dysfunction in neurodegenerative disorders. Prog Neurobiol 2014, 112: 24-49.

[72] Sanjuan MA, Dillon CP, Tait SW, Moshiach S, Dorsey F, Connell $\mathrm{S}$, et al. Toll-like receptor signalling in macrophages links the autophagy pathway to phagocytosis. Nature 2007, 450: 1253-1257.

[73] Benveniste EN. Role of macrophages/microglia in multiple sclerosis and experimental allergic encephalomyelitis. J Mol Med (Berl) 1997, 75: 165-173.

[74] Chastain EM, Duncan DS, Rodgers JM, Miller SD. The role of antigen presenting cells in multiple sclerosis. Biochim Biophys Acta 2011, 1812: 265-274.

[75] Francois A, Terro F, Janet T, Rioux Bilan A, Paccalin M, Page G. Involvement of interleukin-1beta in the autophagic process of microglia: relevance to Alzheimer's disease. J Neuroinflammation 2013, 10: 151.

[76] Dello Russo C, Lisi L, Feinstein DL, Navarra P. mTOR kinase, a key player in the regulation of glial functions: relevance for the therapy of multiple sclerosis. Glia 2013, 61: 301-311.

[77] Dello Russo C, Lisi L, Tringali G, Navarra P. Involvement of mTOR kinase in cytokine-dependent microglial activation and cell proliferation. Biochem Pharmacol 2009, 78: 1242-1251.

[78] Alirezaei M, Kiosses WB, Flynn CT, Brady NR, Fox HS. Disruption of neuronal autophagy by infected microglia results in neurodegeneration. PLoS One 2008, 3: e2906.

[79] Wang S, Li B, Qiao H, Lv X, Liang Q, Shi Z, et al. Autophagyrelated gene Atg5 is essential for astrocyte differentiation in the developing mouse cortex. EMBO Rep 2014, 15: 10531061.

[80] Lee SJ, Cho KS, Koh JY. Oxidative injury triggers autophagy in astrocytes: the role of endogenous zinc. Glia 2009, 57: 1351-1361.

[81] Pastor MD, Garcia-Yebenes I, Fradejas N, Perez-Ortiz JM, Mora-Lee S, Tranque P, et al. mTOR/S6 kinase pathway contributes to astrocyte survival during ischemia. J Biol Chem
2009, 284: 22067-22078.

[82] Cross AH, Manning PT, Keeling RM, Schmidt RE, Misko TP. Peroxynitrite formation within the central nervous system in active multiple sclerosis. J Neuroimmunol 1998, 88: 45-56.

[83] Lisi L, Navarra P, Feinstein DL, Dello Russo C. The mTOR kinase inhibitor rapamycin decreases iNOS mRNA stability in astrocytes. J Neuroinflammation 2011, 8: 1.

[84] Sarkar S, Korolchuk VI, Renna M, Imarisio S, Fleming A, Williams $\mathrm{A}$, et al. Complex inhibitory effects of nitric oxide on autophagy. Mol Cell 2011, 43: 19-32.

[85] Chang A, Nishiyama A, Peterson J, Prineas J, Trapp BD. NG2-positive oligodendrocyte progenitor cells in adult human brain and multiple sclerosis lesions. J Neurosci 2000, 20: 6404-6412.

[86] Wolswijk G. Oligodendrocyte precursor cells in the demyelinated multiple sclerosis spinal cord. Brain 2002, 125: 338-349.

[87] Li W, Tang Y, Fan Z, Meng Y, Yang G, Luo J, et al. Autophagy is involved in oligodendroglial precursor-mediated clearance of amyloid peptide. Mol Neurodegener 2013, 8: 27.

[88] Rangaraju S, Verrier JD, Madorsky I, Nicks J, Dunn WA, Jr., Notterpek L. Rapamycin activates autophagy and improves myelination in explant cultures from neuropathic mice. J Neurosci 2010, 30: 11388-11397.

[89] Meikle L, Pollizzi K, Egnor A, Kramvis I, Lane H, Sahin M, et al. Response of a neuronal model of tuberous sclerosis to mammalian target of rapamycin (mTOR) inhibitors: effects on mTORC1 and Akt signaling lead to improved survival and function. J Neurosci 2008, 28: 5422-5432.

[90] Smith CM, Mayer JA, Duncan ID. Autophagy promotes oligodendrocyte survival and function following dysmyelination in a long-lived myelin mutant. J Neurosci 2013, 33: 8088-8100.

[91] Andersson A, Covacu R, Sunnemark D, Danilov AI, Dal Bianco A, Khademi M, et al. Pivotal advance: HMGB1 expression in active lesions of human and experimental multiple sclerosis. J Leukoc Biol 2008, 84: 1248-1255.

[92] Laudiero LB, Aloe L, Levi-Montalcini R, Buttinelli C, Schilter $D$, Gillessen S, et al. Multiple sclerosis patients express increased levels of beta-nerve growth factor in cerebrospinal fluid. Neurosci Lett 1992, 147: 9-12.

[93] Florez-McClure ML, Linseman DA, Chu CT, Barker PA, Bouchard RJ, Le SS, et al. The p75 neurotrophin receptor can induce autophagy and death of cerebellar Purkinje neurons. J Neurosci 2004, 24: 4498-4509.

[94] Lindberg RL, De Groot CJ, Certa U, Ravid R, Hoffmann $\mathrm{F}$, Kappos L, et al. Multiple sclerosis as a generalized CNS disease--comparative microarray analysis of normal appearing white matter and lesions in secondary progressive MS. J Neuroimmunol 2004, 152: 154-167. 
[95] Kim SR, Chen X, Oo TF, Kareva T, Yarygina O, Wang C, et al. Dopaminergic pathway reconstruction by Akt/Rhebinduced axon regeneration. Ann Neurol 2011, 70: 110-120.

[96] Park KK, Liu K, Hu Y, Smith PD, Wang C, Cai B, et al. Promoting axon regeneration in the adult CNS by modulation of the PTEN/mTOR pathway. Science 2008, 322: 963-966.

[97] Tullman MJ. A review of current and emerging therapeutic strategies in multiple sclerosis. Am J Manag Care 2013, 19:
S21-27.

[98] Zhang Y, Guo TB, Lu H. Promoting remyelination for the treatment of multiple sclerosis: opportunities and challenges. Neurosci Bull 2013, 29: 144-154.

[99] Dang S, Xu H, Xu C, Cai W, Li Q, Cheng Y, et al. Autophagy regulates the therapeutic potential of mesenchymal stem cells in experimental autoimmune encephalomyelitis. Autophagy 2014, 10: 1301-1315. 